\title{
Neutrino-induced reactions on ${ }^{56} \mathrm{Fe}$ and ${ }^{56} \mathrm{Ni}$, and production of ${ }^{55} \mathrm{Mn}$ in population III stars
}

\author{
Toshio Suzuki, ${ }^{1,2}$ Michio Honma, ${ }^{3}$ Koji Higashiyama, ${ }^{4}$ Takashi Yoshida, ${ }^{5}$ Toshitaka Kajino, ${ }^{5,6}$ \\ Takaharu Otsuka, ${ }^{7,8,9}$ Hideyuki Umeda, ${ }^{5}$ and Ken'ichi Nomoto ${ }^{5,10}$ \\ ${ }^{1}$ Department of Physics, College of Humanities and Sciences, Nihon University Sakurajosui 3-25-40, Setagaya-ku, Tokyo 156-8550, Japan \\ ${ }^{2}$ Center for Nuclear Study, University of Tokyo, Hirosawa, Wako-shi, Saitama 351-0198, Japan \\ ${ }^{3}$ Center for Mathematical Sciences, University of Aizu, Aizu-Wakamatsu, Fukushima, Japan \\ ${ }^{4}$ Department of Physics, Chiba Institute of Technology, Narashino, Chiba 275-0023, Japan \\ ${ }^{5}$ Department of Astronomy, Graduate School of Science, University of Tokyo, Bunkyo-ku, Tokyo 113-0033, Japan \\ ${ }^{6}$ National Astronomical Observatory of Japan, Mitaka, Tokyo 181-8588, Japan \\ ${ }^{7}$ Department of Physics, University of Tokyo, Hongo, Bunkyo-ku, Tokyo 113-0033, Japan \\ ${ }^{8}$ Center for Nuclear Study, University of Tokyo, Hongo, Bunkyo-ku, Tokyo 113-0033, Japan \\ ${ }^{9}$ RIKEN, Hirosawa, Wako-shi, Saitama 351-0198, Japan \\ ${ }^{10}$ Institute for the Physics and Mathematics of the Universe, University of Tokyo, Chiba 277-8582, Japan
}

(Received 25 May 2009; published 16 June 2009)

\begin{abstract}
Neutrino-induced reactions on ${ }^{56} \mathrm{Fe}$ and ${ }^{56} \mathrm{Ni}$ are investigated based on a new shell-model Hamiltonian for pf shell. The calculated charged current reaction cross section on ${ }^{56} \mathrm{Fe}$ induced by decay-at-rest (DAR) neutrinos is shown to be consistent with the observation. The Gamow-Teller strength in ${ }^{56} \mathrm{Ni}$ is found to be more spread compared to previous calculations and result in a considerably large branching ratio for the proton knock-out channel. As a consequence of this, the production yields of heavy elements such as ${ }^{55} \mathrm{Mn}$ in population III stars are shown to be enhanced for the new Hamiltonian.
\end{abstract}

DOI: 10.1103/PhysRevC.79.061603

PACS number(s): 25.30.-c, 21.60.Cs, 26.30.-k

Recently, shell-model studies in nuclei have made extensive progress due to the rapid development of computing systems as well as new radioactive facilities. New shell-model Hamiltonians have been invented and used to study structure and transition properties of both stable and unstable nuclei [1-5]. A new shell-model Hamiltonian for the $p$-shell [1], which reproduces Gamow-Teller (GT) transitions in ${ }^{12} \mathrm{C}$ and ${ }^{14} \mathrm{C}$ as well as magnetic moments of $p$-shell nuclei, has been applied to $v$-induced reactions on ${ }^{12} \mathrm{C}$. The reaction cross sections for ${ }^{12} \mathrm{C}$ and ${ }^{4} \mathrm{He}$ were found to be enhanced compared with previous calculations [6]. As a consequence of this, a possible enhancement of the production yields of light elements, ${ }^{7} \mathrm{Li}$ and ${ }^{11} \mathrm{~B}$, during supernova explosion has been pointed out $[6,7]$.

Here, we extend our study to $p f$-shell nuclei and investigate neutrino nucleus reactions on $\mathrm{Fe}$ and $\mathrm{Ni}$ as well as its astrophysical implications. We are particularly interested in ${ }^{56} \mathrm{Ni}$ that is produced most in supernova explosions of massive stars. We study GT transitions in ${ }^{56} \mathrm{Fe}$ and ${ }^{56} \mathrm{Ni}$ by shell-model calculations with the use of a new shell-model Hamiltonian for the $p f$ shell, GXPF1J $[3,8]$. The Hamiltonian reproduces the energy position of the $1^{+}$state of ${ }^{48} \mathrm{Ca}$ and describes well the magnetic dipole $(M 1)$ transition strengths in ${ }^{50} \mathrm{Ti},{ }^{52} \mathrm{Cr}$, and ${ }^{54} \mathrm{Fe}$, as well as the GT strength in ${ }^{58} \mathrm{Ni}[8,9]$.

We show that the distribution of the GT strength in ${ }^{56} \mathrm{Ni}$ is more fragmented and quite different from previous calculations. This difference leads to a large branching ratio for the proton knock-out channel and results in the enhancement of the production yields of ${ }^{55} \mathrm{Mn}$ and ${ }^{59} \mathrm{Co}$ in stars. We first show that the new Hamiltonian can well reproduce the observed $B(\mathrm{GT})$ values in $\mathrm{Fe}$ and $\mathrm{Ni}$ isotopes as well as the reaction cross section for ${ }^{56} \mathrm{Fe}\left(v_{e}, e^{-}\right){ }^{56} \mathrm{Co}$ measured for DAR neutrinos. Then, the reaction cross section for
${ }^{56} \mathrm{Ni}\left(v, v^{\prime} p\right){ }^{55} \mathrm{Co}$ is shown to be considerably enhanced due to the spreading of the GT strength in high-energy region in ${ }^{56} \mathrm{Ni}$. The enhancement of the production yield of ${ }^{55} \mathrm{Mn}$ through the process, ${ }^{55} \mathrm{Co}\left(e^{-}, v_{e}\right){ }^{55} \mathrm{Fe}\left(e^{-}, v_{e}\right){ }^{55} \mathrm{Mn}$, is demonstrated in a population III star.

Shell-model calculations are carried out by using the code MSHELL [10] allowing at most five nucleons to be excited from the $f_{7 / 2}$ orbit into upper orbits relative to the naive filling ground-state configuration. The GT strength distribution can be obtained following the prescription given by Whitehead [11].

Calculated GT strengths for ${ }^{56} \mathrm{Fe}$ are shown in Fig. 1(a). The strengths are suppressed by a universal quenching factor of $(0.74)^{2}$ [12]. The strength for GXPF1J is found to be more fragmented with a remaining tail in the high excitation energy region compared with that obtained by the conventional Hamiltonian, KB3G [13]. This feature is also seen in ${ }^{54} \mathrm{Fe}$ and $\mathrm{Ni}$ isotopes. The calculated sum of the $B\left(\mathrm{GT}_{-}\right)$value in ${ }^{56} \mathrm{Fe}$ is 9.5 (9.0) for GXPF1J (KB3G), which is consistent with the observed value of $9.9 \pm 2.4$ [14], as shown in Table I. Here,

$$
B\left(\mathrm{GT}_{ \pm}\right)=\frac{1}{2 J_{i}+1}\left|\left\langle f \| \sum_{k} f_{q} \sigma_{k} t_{k \pm}|| i\right\rangle\right|^{2},
$$

where $t_{+}|p\rangle=|n\rangle$ and $t_{-}|n\rangle=|p\rangle$ and $f_{q}=0.74$. The calculated sum of the $\mathrm{GT}_{+}$strength in ${ }^{56} \mathrm{Fe}$ is 2.9 (2.5) for GXPF1J $(\mathrm{KB} 3 \mathrm{G})$, which is also close to the experimental value of $2.8 \pm 0.3$ [15]. The calculated and experimental total $B\left(\mathrm{GT}_{ \pm}\right)$ values for ${ }^{54} \mathrm{Fe},{ }^{58} \mathrm{Ni}$, and ${ }^{60} \mathrm{Ni}$ are also shown in Table $\mathrm{I}$. Note that the calculated values in Table I satisfy the GT sum rule; $\sum_{f}\left[B\left(\mathrm{GT}_{-}\right)-B\left(\mathrm{GT}_{+}\right)\right]=3 f_{q}^{2}(N-Z)$. Strengths up to $E_{x}=13 \sim 15 \mathrm{MeV}(8 \sim 10 \mathrm{MeV})$ are included in the experimental values of $B\left(\mathrm{GT}_{-}\right)\left[B\left(\mathrm{GT}_{+}\right)\right]$. The calculated 

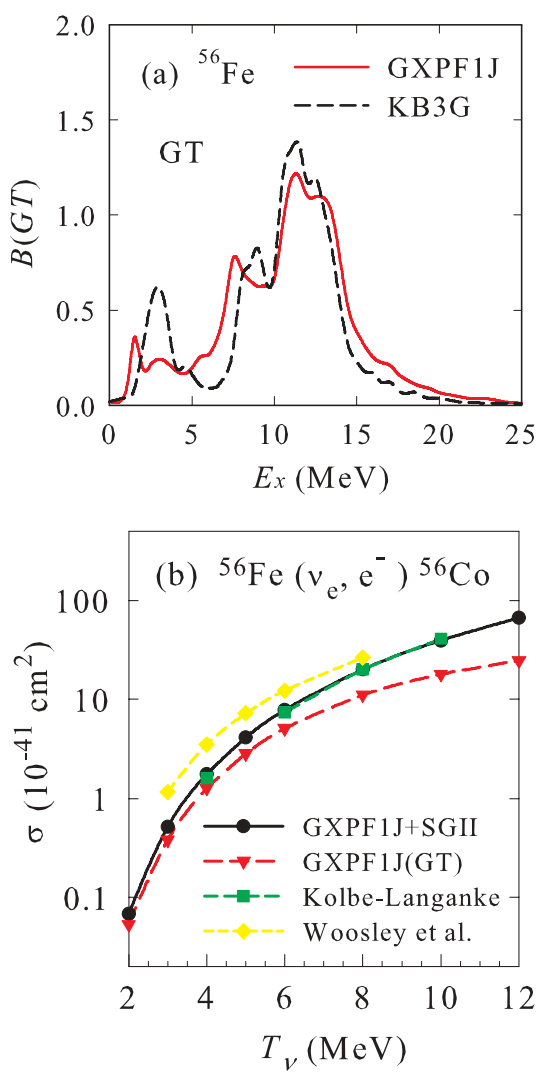

FIG. 1. (Color) (a) GT strengths for ${ }^{56} \mathrm{Fe} \rightarrow{ }^{56} \mathrm{Co}$ obtained for GXPF1J and KB3G Hamiltonians. (b) Cross sections for ${ }^{56} \mathrm{Fe}\left(v_{e}, e^{-}\right){ }^{56} \mathrm{Co}$ induced by supernova neutrinos with temperature $T_{v}$. Results for GT transition obtained for GXPF1J and those including other multipoles obtained by RPA for the SGII interaction are shown. Previous results of Refs. [21-23] are also shown for comparison.

values are rather close to the observed ones. In the case of ${ }^{60} \mathrm{Ni}$, there remains some strength above $E_{x}=15 \mathrm{MeV}$ for GXPF1J, which explains why the calculated $B\left(\mathrm{GT}_{-}\right)$value is larger than the experimental one.

The reaction cross section for ${ }^{56} \mathrm{Fe}\left(v_{e}, e^{-}\right){ }^{56} \mathrm{Co}$ induced by DAR neutrinos is calculated by using the multipole expansion formulas [16] as denoted in Eqs. (4) (7) of Ref. [6]. The GT strength obtained by GXPF1J is used for the evaluation of the axial electric dipole response $\left(T_{1}^{\mathrm{el}, 5}\right)$, the spin part of the $M 1$ response $\left(T_{1}^{\mathrm{mag}}\right)$, as well as the axial Coulomb

TABLE I. Calculated and experimental $B\left(\mathrm{GT}_{-}\right)$and $B\left(\mathrm{GT}_{+}\right)$ values for ${ }^{54,56} \mathrm{Fe}$ and ${ }^{58,60} \mathrm{Ni}$. The GXPF1J Hamiltonian is used for the shell-model calculations with a universal quenching factor $(0.74)^{2}$.

\begin{tabular}{lccccc}
\hline \hline \multirow{2}{*}{ Nucleus } & \multicolumn{2}{c}{$B\left(\mathrm{GT}_{-}\right)$} & & \multicolumn{2}{c}{$B\left(\mathrm{GT}_{+}\right)$} \\
\cline { 2 - 3 } \cline { 6 - 6 } & GXPF1J & EXP. [14] & & GXPF1J & EXP. [15] \\
\hline${ }^{54} \mathrm{Fe}$ & 7.3 & $7.8 \pm 1.9$ & & 4.0 & $3.3 \pm 0.5$ \\
${ }^{56} \mathrm{Fe}$ & 9.5 & $9.9 \pm 2.4$ & & 2.9 & $2.8 \pm 0.3$ \\
${ }^{58} \mathrm{Ni}$ & 8.0 & $7.4 \pm 1.8$ & & 4.7 & $3.8 \pm 0.4$ \\
${ }^{60} \mathrm{Ni}$ & 9.9 & $7.2 \pm 1.8$ & & 3.4 & $3.1 \pm 0.1$ \\
\hline \hline
\end{tabular}

and longitudinal dipole $\left(M_{1}^{5}\right.$ and $\left.L_{1}^{5}\right)$ responses. Effects of finite momentum transfer $q$ are treated by the approximation $j_{0}(q r) \simeq 1-\frac{1}{6} q^{2}\left\langle r^{2}\right\rangle$, where $\left\langle r^{2}\right\rangle$ is taken to be the meansquare charge radius of the nucleus. The terms involving $\vec{\sigma} \cdot \vec{\nabla}$ as well as the orbital part of the $M 1$ response are not taken into account. The transition to the isobaric analog state (IAS) is evaluated also by the shell-model calculations. The cross section for the GT (GT+IAS) transition is calculated to be $\sigma=1.72(2.29) \times 10^{-40} \mathrm{~cm}^{2}$.

Multipoles except for $0^{+}$and $1^{+}$are evaluated by the method of random-phase approximation (RPA) with the use of the SGII interaction [17]. We adopt this hybrid model, as the evaluation of spin-dipole transitions by shell-model calculations is not yet available at present. The same universal quenching for the axial vector coupling constant, $g_{A}^{\text {eff }} / g_{A}=$ 0.74 , is used for all multipoles. Backward electron-scattering data in ${ }^{48} \mathrm{Ca}$ and ${ }^{90} \mathrm{Zr}$ show that the degree of quenching in spin quadrupole $(M 2)$ transitions is very similar to the $M 1$ case [18]. An effective spin $g$ factor $g_{s}^{\text {eff }}=0.64 g_{s}$ is shown to reproduce the $M 2$ strengths in ${ }^{48} \mathrm{Ca}$ and ${ }^{90} \mathrm{Zr}$ as well as the $M 1$ strength in ${ }^{48} \mathrm{Ca}$ [18]. It is therefore reasonable to assume that strengths of spin-dipole transitions in $p f$-shell nuclei are also quenched similar to the GT transition.

The total cross section including all multipoles up to $J=4$ is obtained to be $\sigma=2.69 \times 10^{-40} \mathrm{~cm}^{2}$, which is consistent with the experimental value of the KARMEN Collaboration; $\sigma=2.56 \pm 1.08 \pm 0.43 \times 10^{-40} \mathrm{~cm}^{2}[19]$. The present result is not far from that in Ref. [20], $\sigma=2.38 \times 10^{-40} \mathrm{~cm}^{2}$, where contributions from multipoles except for $1^{+}$are assumed not to be quenched. Note that our result for $1^{+}$is large compared with that in Ref. [20], where only the axial electric dipole response $\left(T_{1}^{\mathrm{el}, 5}\right)$ is considered for the GT transition. The interference term between the $T_{1}^{\mathrm{el}, 5}$ and $M 1\left(T_{1}^{\mathrm{mag}}\right)$ responses included in the present calculation enhances the cross section.

Calculated cross sections for supernova neutrinos with temperature $T_{\nu}$ are shown in Fig. 1(b). The Fermi-Dirac distribution with zero chemical potential is assumed for the neutrino spectra. Results of previous calculations [21-23] are also shown. The present results are similar to those of Ref. [23], slightly larger for $T \leqslant 8 \mathrm{MeV}$ but smaller than those of Ref. [21]. The difference in the GT contribution seems to compensate the difference in the treatment of the quenching in other multipoles, similar to the DAR case. If multipoles other than $1^{+}$are assumed not to be quenched, our results become closer to those of Refs. [21,22].

Now, we discuss neutral current reactions on ${ }^{56} \mathrm{Ni}$. The proton knock out reaction ${ }^{56} \mathrm{Ni}\left(\nu, v^{\prime} p\right){ }^{55} \mathrm{Co}$ is quite interesting as it leads to production of $\mathrm{Mn}$ through electron capture twice; ${ }^{55} \mathrm{Co}\left(e^{-}, v_{e}\right)^{55} \mathrm{Fe}\left(e^{-}, v_{e}\right){ }^{55} \mathrm{Mn}$. The GT transition strengths in ${ }^{56} \mathrm{Ni}\left[B\left(\mathrm{GT}_{0}\right)\right]$ are shown in Fig. 2(a) as a function of excitation energy of ${ }^{56} \mathrm{Ni}$. As the ground state of ${ }^{56} \mathrm{Ni}$ has isospin $T=0$, the $\mathrm{GT}_{0}$ transitions to the $T=1$ states of ${ }^{56} \mathrm{Ni}$ are the same as the GT - transitions to ${ }^{56} \mathrm{Co}$. We find that the strength is fragmented into two peaks for GXPF1J, while it has only one peak for KB3G. This difference in the distribution of the strength leads to different $\left(\nu, v^{\prime} p\right)$ cross sections, though the total $B(\mathrm{GT})$ value does not change much, namely 6.20 and 5.37 for GXPF1J and KB3G, respectively. 

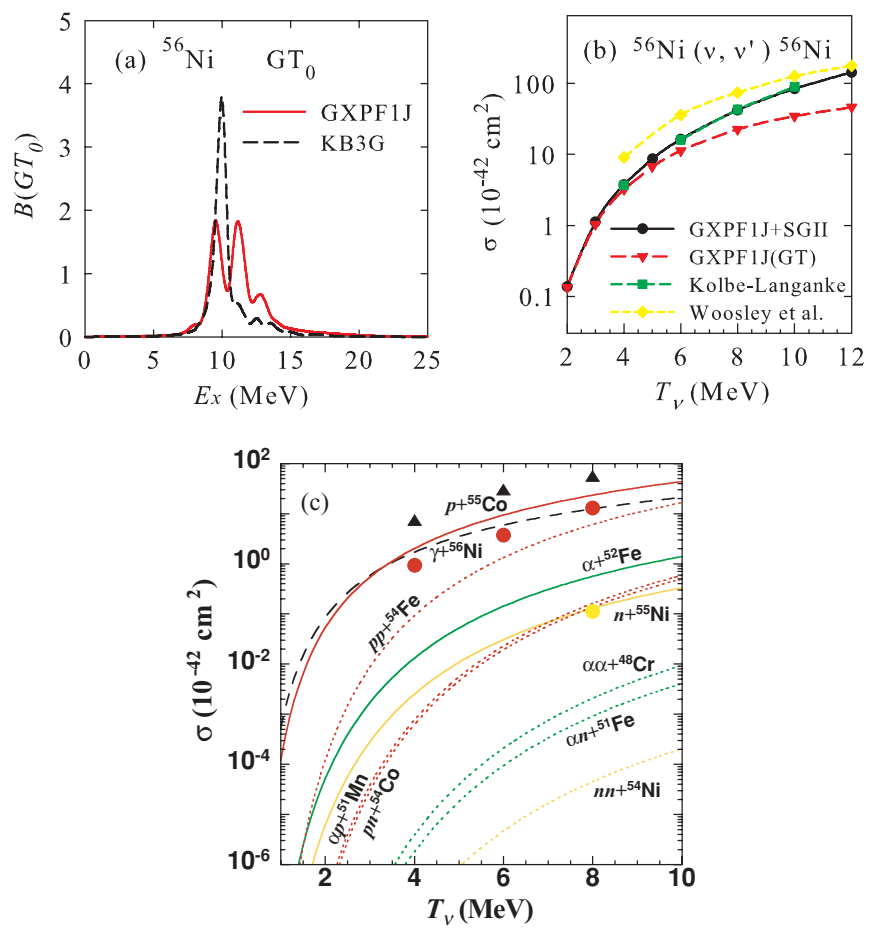

FIG. 2. (Color) (a) Calculated GT strength in ${ }^{56} \mathrm{Ni}$ for GXPF1J and KB3G. (b) The same as in Fig. 1(b) for the neutral current reaction ${ }^{56} \mathrm{Ni}\left(v, v^{\prime}\right){ }^{56} \mathrm{Ni}$ induced by supernova neutrinos with temperature $T_{v}$. The cross sections are averaged over neutrinos and antineutrinos. (c) Cross sections for particle knock-out and $\gamma$ emission channels in ${ }^{56} \mathrm{Ni}\left(\nu, v^{\prime} X\right)$ induced by supernova neutrinos with temperature $T_{v}$. Cases of one and two particle knock-out are shown. Results obtained by using cross sections of HW92 [22] are denoted by triangles, filled red circles, and yellow circles, respectively, for $\gamma$ emission, one proton and one neutron knock-out cases.

The spreading of the $\mathrm{GT}_{0}$ strength is also seen in neutron-rich $\mathrm{Ni}$ isotopes such as ${ }^{58-64} \mathrm{Ni}$. Contributions from multipoles in addition to $1^{+}$are evaluated by the RPA method as for the ${ }^{56} \mathrm{Fe}$ case. The universal quenching, $g_{A}^{\text {eff }} / g_{A}=0.74$, is adopted also here for all multipoles. Calculated cross sections for ${ }^{56} \mathrm{Ni}\left(v, v^{\prime}\right){ }^{56} \mathrm{Ni}$ induced by supernova neutrinos are shown in Fig. 2(b). The contribution from the GT transition is dominant at low temperature, while spin-dipole and other transitions become important at $T \geqslant 8 \mathrm{MeV}$. As in the charged current reaction on ${ }^{56} \mathrm{Fe}$, the present results are close to those of Ref. [23] but small compared to those of Ref. [21].

As the transition to the ground state of ${ }^{55} \mathrm{Co}\left(7 / 2^{-}\right)$is hindered because of the $f$-wave nature of the emitted proton, the strength just above the proton threshold energy, $S_{p}=$ 7.17 MeV, does not contribute to the cross section. The proton emission channel actually opens at $E_{x}=10.1 \mathrm{MeV}$ when the transition to the $1 / 2^{+}(2.92 \mathrm{MeV})$ state of ${ }^{55} \mathrm{Co}$ begins to contribute to the cross section by emitting $s$-wave protons. How much strength exists above $E_{x}=10.1 \mathrm{MeV}$ in ${ }^{56} \mathrm{Ni}$ is essential for the proton knock-out reaction. The calculated strength above $E_{x}=10.1 \mathrm{MeV}$ is 3.83 , that is, $62 \%$ of the total strength, for GXPF1J.

Calculated cross sections for particle knock-out channels as well as $\gamma$ emission channels are shown in Fig. 2(c) for supernova neutrinos. Branching ratios for $\gamma, p, n$, and $\alpha$ emission channels are calculated by using the Hauser-Feshbach theory [24]. Hauser-Feshbach calculations are performed with the code CASCADE [25]. The particle transmission coefficients are generated using the optical model potentials of Perey [26] for protons, Wilmore and Hodgson [27] for neutrons, and Huizenga and Igo [28] for $\alpha$ 's. Level densities for product nuclei are calculated from a formula that is derived from the Fermi gas model [29]. Multiparticle knock-outs of protons, neutrons, and $\alpha$ 's are taken into account. In Fig. 2(c), contributions from one- and two-particle knock-out processes are shown. We find that the $\left(v, v^{\prime} p\right)$ cross section becomes large for GXPF1J compared to the previous calculation using the cross sections of HW92 [22]. However, the $\gamma$ emission cross section becomes smaller. This is due to large branching ratios for the proton knock-out channels for GXPF1J caused by the spreading of the GT distribution.

We now discuss the production yields of elements during supernova explosion for a population III star with mass $15 M_{\odot}$ [30]. The elemental yields of population III star supernovae are compared with observed elemental abundances of low-mass very metal-poor halo stars and extremely metal-poor halo stars. These metal-poor stars are considered to have suffered the pollution of metals from only a few supernovae evolved from population III stars (e.g., Ref. [31]). Therefore, the comparison of the elemental abundances is a good test for the study of supernova nucleosynthesis. Total neutrino energy of $E_{v}=$ $3 \times 10^{53}$ ergs and neutrino temperatures of $\left(T_{v_{e}}, T_{\bar{v}_{e}}, T_{v_{\mu, \tau}}\right)=$ $(4,4,6) \mathrm{MeV}$ [32] are assumed. Cross sections of Ref. [22] are used except for neutral current reactions on ${ }^{56} \mathrm{Ni}$.

The production yields of $\mathrm{Mn}$ and Co produced in complete Si burning are affected by neutrino processes [30]. Table II shows the production yields of $\mathrm{Mn}$ and Co, as well as the logarithmic values of the yield ratios $[X / Y]$, where $[X / Y] \equiv \log \left(N_{X} / N_{Y}\right)-\log \left(N_{X} / N_{Y}\right)_{\odot}$ and, $N_{X}$ and $N_{Y}$ are the abundances of elements $X$ and $Y$, respectively.

An enhancement of the production yield of ${ }^{55} \mathrm{Mn}$ is obtained when the neutrino processes, ${ }^{56} \mathrm{Ni}\left(v, v^{\prime} p\right)$ ${ }^{55} \mathrm{Co}\left(e^{-}, v_{e}\right){ }^{55} \mathrm{Fe}\left(e^{-}, v_{e}\right){ }^{55} \mathrm{Mn}$, are included as shown in Table II. The yield for the GXPF1J Hamiltonian is enhanced compared with those for other Hamiltonians. The calculated values are consistent with the abundances observed in extremely metal-poor stars with $[\mathrm{Fe} / \mathrm{H}] \leqslant-3$ [33]. The proton knocked out from ${ }^{56} \mathrm{Ni}$ by the neutrino-induced reaction enhances the production yield of ${ }^{59} \mathrm{Co}$ through the reaction chain ${ }^{58} \mathrm{Ni}(p, \gamma){ }^{59} \mathrm{Cu}\left(e^{-}, v_{e}\right){ }^{59} \mathrm{Ni}\left(e^{-}, v_{e}\right){ }^{59} \mathrm{Co}$. Enhancement of the production yield of ${ }^{59} \mathrm{Co}$ is also shown in Table II, though the neutrino processes taken into account here are not yet sufficient to explain the observed data. The enhancement of the production yields of $\mathrm{Mn}$ and Co shows the importance of the neutrino processes in nucleosynthesis in stars. It would be quite interesting to carry out more accurate measurements of element abundances as well as more studies on neutrino reaction processes in explosive stars.

Finally, we stress that the origin of the difference of the production yields of the elements is the new GT strength distribution in ${ }^{56} \mathrm{Ni}$ obtained by the new Hamiltonian, which is based on recent extensive studies on both stable and unstable nuclei. 
TABLE II. Production yields of Mn and Co, as well as the logarithmic values of the ratio of these yields relative to Fe, relative to the solar abundances, $[\mathrm{Mn} / \mathrm{Fe}]$ and $[\mathrm{Co} / \mathrm{Fe}]$, in a supernova explosion model of a population III star with $15 M_{\odot}$. See text for details.

\begin{tabular}{lcccrrr}
\hline \hline Model & GXPF1J $(\mathrm{GT})$ & KB3G $(\mathrm{GT})$ & GXPF1J + SGII & HW02 & No $v$ & OBS. [33] \\
\hline$M\left({ }^{55} \mathrm{Co}\right) /\left(10^{-4} M_{\odot}\right)$ & 4.16 & 3.56 & 4.83 & 3.80 & 2.29 & \\
$M\left({ }^{55} \mathrm{Mn}\right) /\left(10^{-4} M_{\odot}\right)$ & 4.31 & 3.72 & 4.99 & 3.96 & 2.30 & $-0.43+0.31 /-0.17$ \\
{$[\mathrm{Mn} / \mathrm{Fe}]$} & -0.25 & -0.32 & -0.19 & -0.29 & -0.53 & 0.08 \\
$M\left({ }^{59} \mathrm{Co}\right) /\left(10^{-5} M_{\odot}\right)$ & 4.47 & 3.53 & 5.77 & 3.64 & -1.10 & $+0.31+0.25 /-0.41$ \\
{$[\mathrm{Co} / \mathrm{Fe}]$} & -0.64 & -0.74 & -0.53 & -0.73 & -10 \\
\hline \hline
\end{tabular}

This work has been supported in part by Grantsin-Aid for Scientific Research (C) 18540290, 20540284, (A) 20244022, Grant-in-Aid for Young Scientists (B) 19740146, Scientific Research on Innovative Areas
(20105001, 20105003), and Global COE Program "the Physical Sciences Frontier," MEXT, Japan. The authors thank Professor W. Bentz for careful reading of the manuscript.
[1] T. Suzuki, R. Fujimoto, and T. Otsuka, Phys. Rev. C 67, 044302 (2003).

[2] Y. Utsuno, T. Otsuka, T. Mizusaki, and M. Honma, Phys. Rev. C 60, 054315 (1999).

[3] M. Honma, T. Otsuka, B. A. Brown, and T. Mizusaki, Phys. Rev. C 65, 061301(R) (2002); 69, 034335 (2004).

[4] T. Otsuka, M. Honma, T. Mizusaki, N. Shimizu, and Y. Utsuno, Prog. Part. Nucl. Phys. 47, 319 (2001).

[5] T. Suzuki and T. Otsuka, Phys. Rev. C 78, 061302(R) (2008).

[6] T. Suzuki, S. Chiba, T. Yoshida, T. Kajino, and T. Otsuka, Phys. Rev. C 74, 034307 (2006).

[7] T. Yoshida, T. Suzuki, S. Chiba, T. Kajino, H. Yokomakura, K. Kimura, A. Takamura, and D. H. Hartmann, Astrophys. J. 686, 448 (2008).

[8] M. Honma, T. Otsuka, T. Mizusaki, M. Hjorth-Jensen, and B. A. Brown, J. Phys. Conf. Ser. 20, 7 (2005).

[9] Y. Fujita et al., Eur. Phys. J. A 13, 411 (2002).

[10] T. Mizusaki, RIKEN Accel. Prog. Rep. 33, 14 (2000).

[11] R. R. Whitehead, in Moment Methods in Many-Fermion Systems, edited by B. J. Dalton et al. (Plenum, New York, 1980), p. 235.

[12] G. Martínez-Pinedo, A. Poves, E. Caurier, and A. P. Zuker, Phys. Rev. C 53, R2602 (1996).

[13] A. Poves, J. Sánchez-Solano, E. Caurier, and F. Nowacki, Nucl. Phys. A694, 157 (2001); E. Caurier, G. Martínez-Pinedo, F. Nowacki, A. Poves, and A. P. Zuker, Rev. Mod. Phys. 77, 427 (2005).

[14] J. Rapaport et al., Nucl. Phys. A410, 371 (1983).

[15] E. Caurier, K. Langanke, G. Martínez-Pinedo, and F. Nowacki, Nucl. Phys. A653, 439 (1999).

[16] J. D. Walecka, in Muon Physics, edited by V. H. Highes and C. S. Wu (Academic Press, New York, 1975), Vol. II; J. S.
O'Connell, T. W. Donnelly, and J. D. Walecka, Phys. Rev. C 6, 719 (1972); T. W. Donnelly and J. D. Walecka, Nucl. Phys. A274, 368 (1976); T. W. Donnelyy and W. C. Haxton, At. Data Nucl. Data Tables 23, 103 (1979).

[17] Nguyen Van Giai and H. Sagawa, Phys. Lett. B106, 379 (1981).

[18] P. von Neumann-Cosel, F. Neumeyer, S. Nishizaki, V. Yu. Ponomarev, C. Rangacharyulu, B. Reitz, A. Richter, G. Schrieder, D. I. Sober, T. Waindzoch, and J. Wambach, Phys. Rev. Lett. 82, 1105 (1999).

[19] R. Maschuw et al., Prog. Part. Nucl. Phys. 40, 183 (1998).

[20] E. Kolbe, K. Langanke, and G. Martínez-Pinedo, Phys. Rev. C 60, 052801(R) (1999).

[21] S. E. Woosley, D. H. Hartmann, R. D. Hoffman, and W. C. Haxton, Astrophys. J. 356, 272 (1990).

[22] R. D. Hoffman and S. E. Woosley, Neutrino Interaction Cross Sections and Branching Ratios, http://adg.llnl.gov/ Research/RRSN/nu_csbr/neu_rate.html (1992).

[23] E. Kolbe and K. Langanke, Phys. Rev. C 63, 025802 (2001).

[24] W. Hauser and H. Feshbach, Phys. Rev. 87, 366 (1952).

[25] F. Pühlhofer, Nucl. Phys. A280, 267 (1977).

[26] F. G. Perey, Phys. Rev. 131, 745 (1963).

[27] D. Wilmore and P. E. Hodgson, Nucl. Phys. 55, 673 (1964).

[28] J. R. Huizenga and G. Igo, Nucl. Phys. 29, 462 (1962).

[29] W. Dilg, W. Schantl, H. Vonach, and M. Uhl, Nucl. Phys. A217, 269 (1973).

[30] T. Yoshida, H. Umeda, and K. Nomoto, Astrophys. J. 672, 1043 (2008).

[31] T. Shigeyama and T. Tsujimoto, Astrophys. J. 507, L135 (1998).

[32] T. Yoshida, H. Umeda, and K. Nomoto, Astrophys. J. 631, 1039 (2005).

[33] Cayrel et al., Astron. Astrophys. 416, 1117 (2004). 